\title{
Targeting Regional Nodal Basins in Breast Cancer Patients: Exactly What Are We Treating?
}

\author{
Sharad Goyal, MD and Bruce G. Haffty, MD \\ Department of Radiation Oncology, Rutgers Cancer Institute of New Jersey, Rutgers Robert Wood Johnson Medical \\ School, New Brunswick, NJ
}

The American College of Surgeons Oncology Group (ACOSOG) Z0011 trial, a phase III non-inferiority trial sought to determine the effects of an axillary lymph node dissection (ALND) on overall survival (OS) and diseasefree survival (DFS) in patients with one to two positive sentinel lymph nodes (SLNs) from breast cancer. ${ }^{1}$ Patients with clinical T1-T2 invasive breast cancer, no palpable adenopathy, and one to two SLNs containing metastases were randomized to ALND or no further axillary evaluation; all patients subsequently received whole-breast irradiation (WBI). In this group of patients, no significant difference in OS or DFS was observed after ALND. While considered a practice-changing study, the ACOSOG Z0011 study has several limitations, including the following: the study closed early with less than $50 \%$ of the targeted accrual; there were lower-than-expected event rates; and there was a high proportion of patients lost to follow-up (21\% in the ALND group and $17 \%$ in the SLN group). In addition, from a radiation oncologist perspective, while all patients had to undergo WBI with opposing standard tangential fields (i.e. no third-field irradiation allowed), there was no radiotherapy (RT) quality assurance and field design was not uniform. In fact, a post hoc analysis of the ACOSOG Z0011 study was performed in an attempt to quantify whether the regional nodes were treated. ${ }^{2}$ Detailed radiation treatment records for 228 patients were submitted for post hoc central review. It was determined that high tangents were used in up to $50 \%$ of patients and that $19 \%$ had received directed regional nodal $\mathrm{RT}$ using $\geq 3$ fields.

(C) Society of Surgical Oncology 2014

First Received: 15 July 2014;

Published Online: 1 August 2014

B. G. Haffty, MD

e-mail: hafftybg@cinj.rutgers.edu
The benefit of regional nodal irradiation (RNI) in patients with a positive axilla has been demonstrated in several large clinical trials. The EORTC 22922-10925 randomized patients with an involved axilla and/or medial primary tumor to $\mathrm{WBI} \pm$ internal mammary and medial supraclavicular LN (IM-MS) after lumpectomy and ALND. ${ }^{3}$ After enrolling over 4,000 women with a followup of 10.9 years, it was reported that IM-MS irradiation improved OS, DFS, and metastases-free survival without an increase in non-breast cancer-related mortality. Similarly, the NCIC MA-20 randomized 1,832 women with high-risk node-negative or node-positive breast cancer to $\mathrm{WBI} \pm \mathrm{RNI}$ (internal mammary, supraclavicular, and high axillary lymph nodes) and lumpectomy and ALND. ${ }^{4}$ With a median follow-up of 5 years, the authors reported that RNI offered women a statistically significant benefit in terms of locoregional DFS, distant DFS and OS with acceptable rates of toxicities. In both the EORTC 22922-10925 and NCIC MA-20, the therapeutic benefit of nodal irradiation in breast cancer patients who receive ALND is established and thus raises the question of whether the axilla needs to receive a tumorcidal dose after positive SLN biopsy (SLNB) alone. Thus, the EORTC 10981/22023 AMAROS (after mapping of the axilla: radiotherapy or surgery) trial sought to answer this question by randomizing 1,425 women with a positive SLNB to ALND or axillary RT. ${ }^{5}$ With a median follow-up of 6.1 years, the 5-year axillary recurrence rate after a positive SNB was $0.54 \%$ (4/744) after ALND and $1.03 \%$ (7/ 681) after axillary RT ( $p=$ not significant), with no difference in OS and DFS. However, ALND caused a twofold higher incidence of lymphedema compared with ART $(p<0.0001)$.

In this issue of Annals of Surgical Oncology, Belkacemi and colleagues report a prospective study of titanium clip placement in the region of the excised SLNs and how this 
region is effectively covered with WBI. This prospective study included 25 patients who underwent breast-conserving surgery and SLNB procedure for invasive breast cancer. SLNB mapping was performed using technetium$99 \mathrm{~m}$-labeled human albumin colloid particles and/or blue dye, and, after the SLNs were identified and removed, two titanium clips were placed to mark the location. Subsequently, all patients underwent computed tomography (CT) simulation and WBI, which was defined individually to target the whole-breast (WB) volume. The standard WB volume was defined with the superior border set at $2 \mathrm{~cm}$ below the humeral head, while a more generous WB volume (high tangents) was defined with a superior border placed at the inferior edge of the humeral head. RT dosevolume histograms were then analyzed according to axilla volumes. The authors report that the average doses using standard WBI delivered to the nodal regions were axillary levels I (25 Gy), II (5 Gy), III (2 Gy) and SLNB (33 Gy). This was significantly improved when using WBI delivered with high tangents: level 1 (38 Gy), level II (11 Gy), level III (5 Gy) and SLNB (45 Gy), with a $p$ value $<0.02$. It should be noted that there was no statistically significant difference in lung dose, while in left-sided patients, the mean heart dose was noted to be higher with high tangents compared with standard tangents $(2.6$ vs. $1.4 \mathrm{~Gy}$; $p=0.02$ ).

There are several implications for this study. First, it was interesting to note that there was significant variation of the anatomical location of the SLNs among patients, thus preventing a uniform field design for breast cancer patients. This emphasizes the need for CT-based treatment planning to document the degree of nodal coverage. This study also revealed that when using standard WBI fields, the region of the SLNs, as identified by targeted clip placement, is underdosed and that the use of high tangents improved the dose coverage from 40 to $80 \%$ of patients. While minimization of surgical interventions has improved the quality of life of breast cancer patients without affecting disease outcomes, it has also put greater importance on the utility of RT in this patient population. In the era of Z0011, radiation oncologists should be cognizant of when to apply RNI and coverage of the axilla in this patient population. With careful attention to surgical technique and pathologic details in addition to the proper utilization of RT field design, we can help ensure that local relapse and toxicity rates in the conservatively managed breast cancer patient continues to be low. In addition, one may refer to nomograms which predict the risk of having additional non-SLN in patients with a positive SLNB when determining the optimal field design for patients who did not receive an ALND. Finally, efforts should be made to determine the optimal management of the regional nodes in patients with positive SLNB in a multidisciplinary setting.

CONFLICT OF INTEREST Sharad Goyal and Bruce G. Haffty have no conflicts of interest to report.

\section{REFERENCES}

1. Giuliano AE, et al. Axillary dissection vs no axillary dissection in women with invasive breast cancer and sentinel node metastasis: a randomized clinical trial. JAMA. 2011;305(6):569-75.

2. Jagsi R, et al. Radiation field design on the ACOSOG Z0011 trial. San Antonio Breast Cancer Symposium 2013: San Antonio, TX.

3. Poortmans $\mathrm{P}$, et al. Irradiation of the internal mammary and medial supraclavicular lymph nodes in stage I to III breast cancer: 10-year results of the EORTC Radiation Oncology and Breast Cancer Groups phase III trial. European Cancer Congress, 2013. Amsterdam.

4. Whelan T, et al. NCIC-CTG MA.20: an intergroup trial of regional nodal irradiation in early breast cancer. In: American Society of Clinical Oncology Annual Meeting, 2011.

5. Rutgers E, et al. Radiotherapy or surgery of the axilla after a positive sentinel node in breast cancer patients: final analysis of the EORTC AMAROS trial (10981/22023). In: American Society of Clinical Oncology Annual Meeting, 2013. Chicago, IL. 